\section{Effect of DNA copy number on genetic stability of phage-displayed peptides}

\author{
George P. Smith and Ana-María Fernández \\ University of Missouri, Columbia, MO, USA
}

BioTechniques 36:610-618 (April 2004)

A small model peptide, the FLAG ${ }^{\circledR}$ epitope, was cloned into two filamentous phage display vectors, f88-4 and fd88-4, creating phages f88-FLAG and fd88-FLAG, respectively. Both vectors have a gene VIII display cassette (in addition to their normal phage gene VIII) and display the cloned peptide on a few percent of the virion's 3000-4000 pVIII (major coat protein) subunits. Vector f88-4 has a replication defect and attains low DNA copy number in infected cells, while vector fd88-4 has no replication defect and attains the normal, high DNA copy number characteristic of wild-type filamentous phage. Almost no loss of displayed peptide was observed during six rounds of propagation of low copy number f88-FLAG phage. In contrast, when high copy number fd88-FLAG phage was similarly propagated, variant clones that did not display the FLAG epitope accumulated gradually. The loss of displayed peptide from the high copy number vector is undoubtedly slow enough to be overcome by even weak affinity selection, and high copy number vectors have important advantages that make their use worth considering, at least when the displayed peptides are small.

\section{INTRODUCTION}

Filamentous bacteriophage, the basis of most phage display constructs (1), contains a circular single-stranded DNA (ssDNA) encased in a tubular capsid composed primarily of thousands of copies of the major coat protein pVIII. They infect strains of Escherichia coli that harbor the conjugative $\mathrm{F}$ episome and therefore display the $\mathrm{F}$ pilus on their surface. The pilus mediates the infection process, which culminates in the penetration of the viral ssDNA (the plus strand) into the cell, where a complementary DNA strand (the minus strand) is synthesized by host polymerases to form the doublestranded replicative form (RF). Minusstrand synthesis is initiated with high efficiency by host RNA polymerase at a special minus-strand origin in the intergenic region (2) of the plus strand but can occur at low efficiency in the absence of the minus-strand origin (3). Rolling-circle replication of the double-stranded RF produces progeny plus strands, a process that requires the phage replication protein pII acting at a plus-strand origin that also lies in the intergenic region. Early in infection, progeny plus strands serve as a template for minus-strand synthesis. Balanced plus- and minus-strand syn- thesis thus results in the accumulation of double-stranded RF molecules to a copy number of 100 or more. Late in infection and in chronically infected cells, however, the phage ssDNA-binding protein $\mathrm{pV}$ sequesters nearly all progeny plus strands into a filament-shaped complex. These ssDNAs are extruded through the cell envelope, concomitantly shedding $\mathrm{pV}$ and acquiring the virion coat proteins, including pVIII, from the inner membrane to emerge as completed virions. The extrusion of progeny virions does not kill the cell; chronically infected cells continue to divide, although at a slower rate than uninfected cells. It is the slowing of cell division, not cell lysis, that explains plaque formation by these phage.

Both filamentous phage-display vectors used in this work are of type 88 , meaning they have two copies of gene VIII (encoding pVIII). One is the normal, wild-type gene, and it encodes most of the virion's thousands of major coat protein subunits. The other is a gene VIII display cassette (Figure 1) that has restriction sites that allow foreign peptide coding sequences to be fused to the gene VIII coding sequence. The bottom part of Figure 1 shows how the coding sequence for the FLAG ${ }^{\circledR}$ peptide is cloned into these sites to create a fusion protein with the FLAG peptide at the $\mathrm{N}$ terminus of the mature form of the coat protein (after cleavage by signal peptidase). The display cassette is expressed from an isopropyl- $\beta$ D-thiogalactopyranoside (IPTG)-inducible tac promoter operator; under fully induced conditions, approximately 5\% of the pVIII subunits derive from the cassette and display the foreign peptide.

One of the type 88 vectors used in the present study, f88-4, is derived from phage fd-tet (4), which has a replication defect and therefore reaches low DNA copy number in infected (or transfected) host cells. As diagrammed in the upper part of Figure 2, the replication defect results from a disruption of the minus-strand origin by a long (2.8$\mathrm{kb})$ tetracycline-resistant determinant (Tet), and the gene VIII display cassette replaces a few hundred noncoding base pairs within the determinant. Plaques formed by fd-tet derivatives are so small that it is impractical to quantify infection in terms of plaque-forming units (pfu). However, because infection transduces the infected cell to tetracycline resistance, infectious units can be effectively quantified as transducing units by spreading infected cells on tetracycline-containing nutrient agar and counting colonies. These phages can be propagated independently of infection, even in an uninfectable $\mathrm{F}^{-}$host, by culturing the phage-bearing cells in medium containing the antibiotic. The replication defect reduces infectivity (transducing units per physical particle) about 10-fold relative to wild-type phage (typical infectivity of $0.5 \mathrm{pfu} /$ particle). That is because minus-strand synthesis is greatly delayed in a newly infected cell, thus delaying the expression of the tetracycline-resistant determinant and subjecting the unpaired plus strand to degradation. When the cells are finally challenged with a high concentration of the antibiotic on agar plates or in liquid medium, only a small minority survives. The yield of physical particles in fd-tet-derived vectors such as f88-4 is also reduced to about $25 \%$ of normal levels. The net effect of low infectivity and low particle yield is an overall 40-fold reduction in the yield of infective units when compared with wild-type high copy number phage. 
The obvious disadvantages of the replication defect in f88-4 and other fdtet-derived vectors are offset by a key advantage: it largely averts a complication called "cell killing." When phage assembly is fully or partially blocked, intracellular phage DNA and gene products accumulate to toxic levels, and the host cell is killed without releasing progeny phage (5). Cell killing is nearly absent in fd-tet derivatives such as f88-4 because of their low RF copy number, and even severe morphogenetic defects are readily tolerated (6). Display vectors based on fd-tet therefore accommodate recombinant coat proteins that partially impair phage assembly or that are directly toxic in their own right. Because there is less selection pressure against foreign peptides, we expect displayed peptides to be more stable genetically in low copy number than in high copy number vectors.

The low copy number vector f884 has already been used extensively for both random and natural peptide libraries (7-9). But is it necessary to incur the disadvantages of a low copy number vector, especially when the displayed peptides are short? To address this question, we constructed a high copy number type 88 vector, fd88-4, which has the same gene VIII display cassette as f88-4. As shown in the lower part of Figure 2, the cassette is inserted into a nonessential part of the intergenic region of wild-type $\mathrm{fd}$, where it does not disrupt either the minus-strand origin or the nearby stem loop that serves as a packaging signal during the extrusion of progeny virions. A short model peptide, the FLAG epitope recognized by commercially available monoclonal antibodies, was cloned into both the high and low copy number vectors as diagrammed in the lower part of Figure 1, creating clones fd88-FLAG and f88-FLAG, respectively. Each construct was subjected to six rounds of propagation without selection in favor of the FLAG epitope. Then, after the final round of nonselective propagation, several dozen individual phage subclones were tested for retention of the FLAG epitope. Most, but not all, of the fd88-FLAG subclones had lost the displayed peptide, while all but one of the 888-FLAG subclones retained it. These results therefore cor- roborate the supposition that displayed peptides are more genetically stable in low copy number vectors than in high copy number vectors. At the same time, the rate of epitope loss from the high copy number construct is not so high as to make fd88-4 unappealing as a vector for small peptide libraries.

\section{MATERIALS AND METHODS}

\section{Solutions and Media}

Tris-buffered saline (TBS) is $50 \mathrm{mM}$ Tris-HCl, pH 7.5, 0.15 M NaCl. TBST is TBS with $0.5 \%(\mathrm{v} / \mathrm{v})$ Tween ${ }^{\circledR} 20$. Dialyzed bovine serum albumin (BSA; Sigma, St. Louis, MO, USA) was dissolved at $50 \mathrm{mg} / \mathrm{mL}$ in water and filter sterilized. TTDB is TBST supplemented with $1 \mathrm{mg} / \mathrm{mL}$ dialyzed BSA. TBS/gelatin is TBS supplemented with $1 \mathrm{mg} / \mathrm{mL}$ gelatin and autoclaved. NZY and NZY/Tet liquid medium and nutrient agar Petri dishes have been previously described (10). Soft agar contains $1 \mathrm{~g} \mathrm{Bacto}^{\mathrm{TM}}$ tryptone (Fisher Scientific, St. Louis, MO, USA), $0.5 \mathrm{~g} \mathrm{NaCl}$, and $0.75 \mathrm{~g}$ Bacto agar (Fisher Scientific) per $100 \mathrm{~mL}$ and is stored at room temperature after autoclaving and melted in a microwave oven before use. X-gal stock solution contains $2 \% \mathrm{X}$-gal in dimethyl formamide and is stored at $-20^{\circ} \mathrm{C}$, protected from light.

\section{Phage Constructs and $E$. coli Bacterial Host Strains}

All constructions were accomplished by standard recombinant DNA techniques and confirmed by DNA sequencing. The low copy number vector f88-4 has been previously described (GenBank $^{\circledR}$ accession no. AF218363). The high copy number vector fd88-4 was constructed by inserting the gene VIII display cassette (upper part of Figure 1) into the wild-type phage fd. Starting at fd position 5605 (GenBank accession no. J02451), the plus-strand sequence immediately preceding the first position of the cassette is $5^{\prime}$-GCCACGTT-3'. Ending at fd position 5624 , the plus-strand sequence immediately following the last position of the cassette is $5^{\prime}$-CGGCTTTCC-3'. The coding sequence for the FLAG peptide 
was installed in both vectors, as shown in the lower part of Figure 1.

E. coli K-12 host strains MC1061 $\left[\mathrm{F}^{-}\right.$hsdR mcrB $\Delta($ araABC-leu $) 6779$ araD139 $\Delta$ lac174 galU galK strA thi] and K91 (Hfr Cavalli thi) have been previously described (10). K91 deploys the $\mathrm{F}$ pilus and is therefore infectible by filamentous phage. MC1061 has no $\mathrm{F}$ pilus and is uninfectible but supports phage production at normal levels.

\section{Titering Plaque-Forming and Transducing Units}

The nonreplication-defective phage fd88-4 and fd88-FLAG were titered for blue plaques as follows. Phages were diluted to a suitable range of concentrations in TBS/gelatin, and por- tions of $25-400 \mu \mathrm{L}$ were pipeted into sterile $15-\mathrm{mL}$ polypropylene tubes (Fisherbrand $^{\circledR}$; Fisher Scientific). To each tube, we added $400 \mu \mathrm{L}$ of a late log-phase culture of K91 cells and approximately $3 \mathrm{~mL}$ molten soft agar at $50^{\circ} \mathrm{C}$ premixed with $60 \mu \mathrm{L} 2 \% \mathrm{X}$-gal. Immediately after the addition of the molten agar, the contents of each tube were poured onto an NZY agar plate, which was tipped and rocked to distribute the mixture evenly over the entire surface. After the soft agar had gelled, the plates were incubated overnight at $37^{\circ} \mathrm{C}$. Plaques are visible within about $10 \mathrm{~h}$, and blue color is well-developed in positive clones 6-10 h later. Phage variants that have lost a functional lac operator give white (i.e., naturally colored) plaques under these circumstanc- es (see Results and Discussion).

Tetracycline transducing units for the replication-defective phage, such as f88-4 and f88-FLAG, were titered on K91 cells as previously described (10). Each transducing unit is manifested as a colony of phage-bearing tetracyclineresistant cells on agar medium containing that antibiotic.

\section{Nonselective Propagation of f88-FLAG}

Two well-separated colonies of $E$. coli strain MC1061 cells bearing phage f88-FLAG (about $10^{7}$ virions/colony) were used to inoculate $35 \mathrm{~mL}$ of NZY/ Tet medium. After shaking overnight at $37^{\circ} \mathrm{C}$, the cells were cleared at $4^{\circ} \mathrm{C}$ by two successive 10 -min centrifuga-

\section{Gene VIII display cassette}

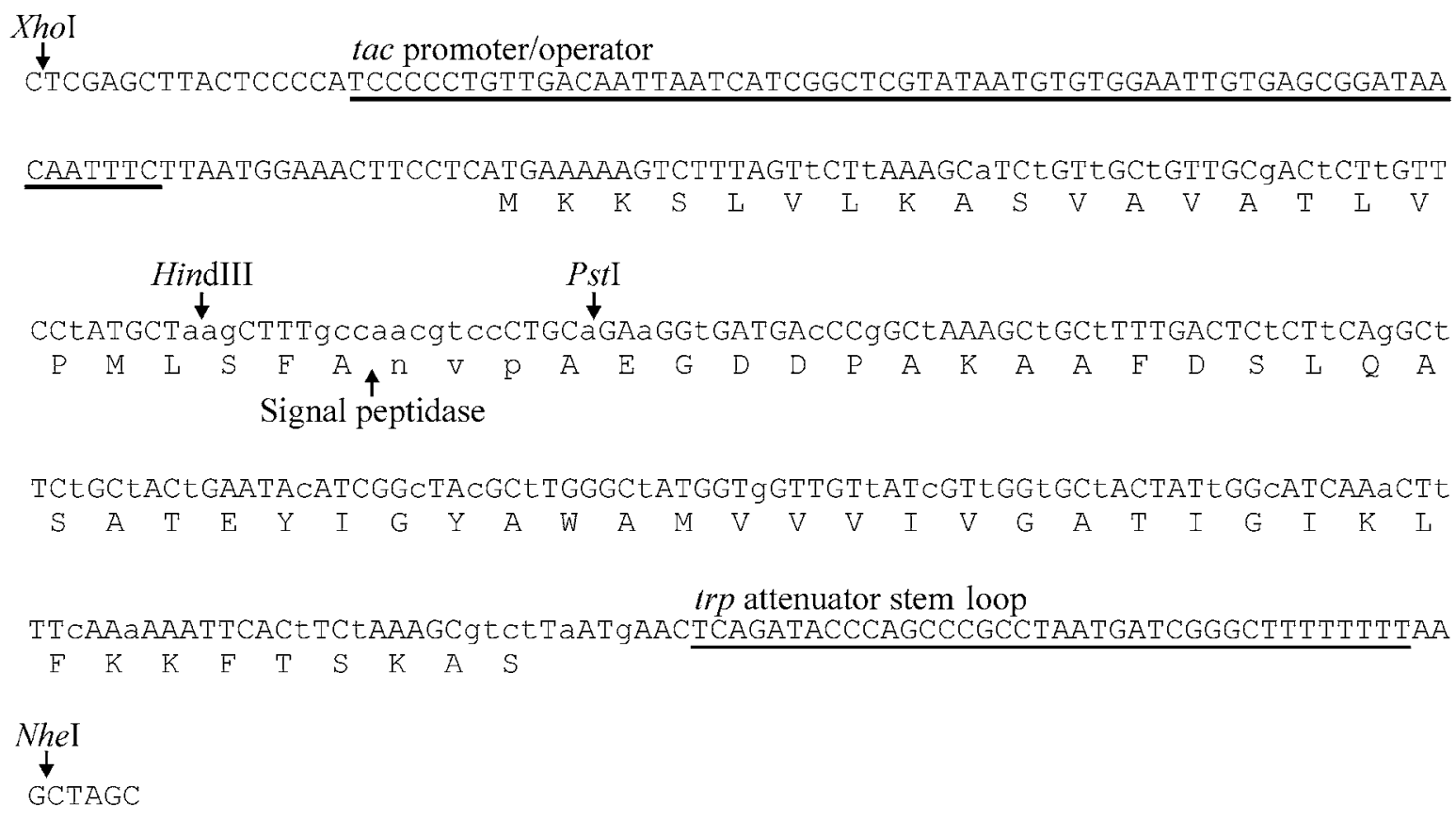

\section{FLAG insert in gene VIII display cassette}

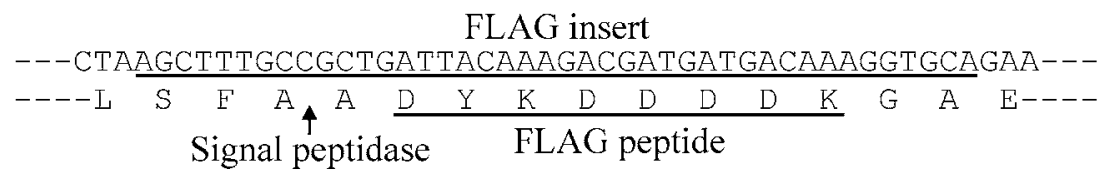

Figure 1. The gene VIII display cassette in f88-4 and fd88-4 vectors (upper part) and the FLAG insert ligated into the HindIII and PstI cloning sites in the cassette (lower part). In the nucleotide and amino acid sequences in the upper part of the figure, residues that differ from those in the wild-type gene VIII or the wild-type pVIII polypeptide are shown in lowercase. In both parts, the peptide bond in the pVIII preprotein polypeptide where signal peptidase cleaves to release the mature protein is indicated. 
tions at $3000 \times$ and $7600 \times \mathrm{g}$. Virions were precipitated from the resulting culture supernatant by two successive polyethylene glycol (PEG) precipitations as previously described (10). A portion of each clone containing about $1.6 \times 10^{10}$ virions was used to infect $500 \mu \mathrm{L}$ of an early log-phase NZY culture of E. coli strain K91 for $10 \mathrm{~min}$ at room temperature, and $400 \mu \mathrm{L}$ of the infected cells were diluted into $20 \mathrm{~mL}$ NZY medium supplemented with 0.2 $\mu \mathrm{g} / \mathrm{mL}$ tetracycline (a subinhibitory concentration that suffices to induce expression of the tetracycline resistance gene) in a $125-\mathrm{mL}$ culture flask that was shaken vigorously at $37^{\circ} \mathrm{C}$ for $1 \mathrm{~h}$. Tetracycline was added to a final concentration of $20 \mu \mathrm{g} / \mathrm{mL}$ (fully inhibitory) and IPTG to a final concentration of $1 \mathrm{mM}$, and shaking was continued overnight at $37^{\circ} \mathrm{C}$. Culture supernatants were cleared of cells by centrifuging at $15,000 \times g$ small volumes of culture for $1 \mathrm{~min}$ in a microcentrifuge. The propagation cycle described in the previous sentence was repeated four more times, except that the phage inoculum was $1 \mu \mathrm{L}$ of culture supernatant from the previous propagation cycle (about 5

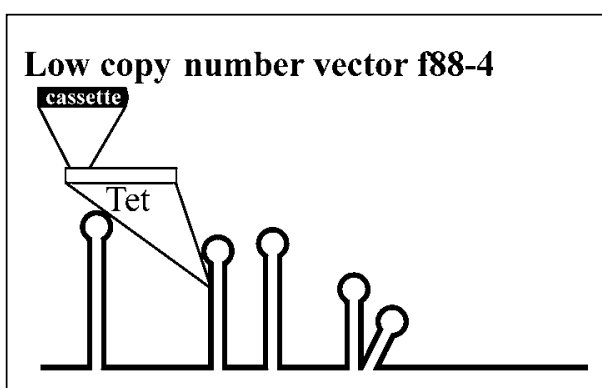

High copy number vector fd88-4

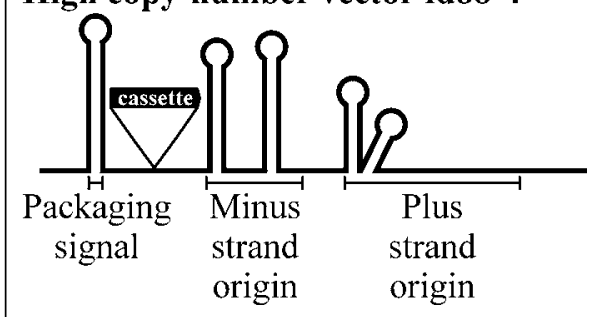

Figure 2. Intergenic region of the filamentous phage genome showing positions of the gene VIII display cassette in the f88-4 and fd88-4 vectors. The three known functional elements of the intergenic region are delineated in the lower part. The tetracycline-resistant determinant (Tet) in f88-4 is $2.8 \mathrm{~kb}$ long and disrupts the minus-strand origin; the gene VIII display cassette replaces a few hundred noncoding base pairs of the determinant. $\times 10^{8}$ virions). The final culture supernatants contained virions that had been propagated nonselectively (i.e., without regard to retention of an intact gene VIII display cassette) for a total of six cycles. The phage amplification factor for the first propagation cycle (from the single colonies) was approximately 1.75 million, for the second cycle about 800 , and for the remaining four cycles about 25,000.

\section{Nonselective Propagation of fd88-FLAG}

Two single blue fd88-FLAG plaques (about $10^{7}$ virions/plaque) were used to inoculate $35-\mathrm{mL}$ portions of an early log-phase culture (about $10^{8}$ cells $/ \mathrm{mL}$ ) of E. coli strain K91 in NZY medium, and after shaking overnight at $37^{\circ} \mathrm{C}$, the virions were prepared by PEG precipitation from cleared culture supernatant as described in the previous subsection. A portion of each clone containing about $7 \times 10^{10}$ virions was used to inoculate $20 \mathrm{~mL}$ of an early log-phase K91 culture that was shaken overnight at $37^{\circ} \mathrm{C}$, and culture supernatants were cleared of cells by centrifuging at

$15,000 \times g$ small volumes of culture for $1 \mathrm{~min}$ in a microcentrifuge. The propagation cycle described in the previous sentence was repeated four more times, except that the phage inoculum was $2 \mu \mathrm{L}$ of culture supernatant from the previous propagation cycle (approximately $4 \times 10^{9}$ virions). The final culture supernatants contained virions that had been propagated nonselectively for a total of six cycles. The phage amplification factor for the first propagation cycle (from the single plaques) was about 7 million, for the second cycle about 570, and for the remaining four cycles about 10,000 .

\section{Testing Clones for Retention of FLAG Epitope}

Suitable dilutions of the final f88-FLAG culture supernatants were titered for tranducing units on K91 host cells as described above. Forty-nine well-separated colonies from each clone were picked to grid points on an NZY/Tet agar dish containing $1 \mathrm{mM}$ IPTG (to induce the 
display cassette) with sterile wooden sticks, as were 11 negative control clones harboring a phage that does not display the FLAG epitope. After overnight growth of the gridded clones at $37^{\circ} \mathrm{C}$, the Petri dish was cooled and blotted with a disk of nitrocellulose. The blot was washed vigorously in tap water to remove all adherent cells, blocked for $1 \mathrm{~h}$ at room temperature in $10 \mathrm{mg} / \mathrm{mL}$ dialyzed BSA in TBS, reacted for $3 \mathrm{~h}$ at room temperature in 5 $\mu \mathrm{g} / \mathrm{mL}$ biotinylated mouse monoclonal anti-FLAG antibody M2 (Sigma) in TTDB, washed extensively with TBST, reacted for $30 \mathrm{~min}$ in $2 \mu \mathrm{g} / \mathrm{mL}$ alkaline phosphatase conjugate of streptavidin (Jackson ImmunoResearch, West Grove, PA, USA), washed extensively in TBST, and developed for a few minutes in 1-Step ${ }^{\mathrm{TM}}$ BCIP/NBT substrate solution (Pierce Chemical, Rockford, IL, USA) until positive purple spots were well-developed. All but one of the 98 f88-FLAG clones were strongly positive. All 11 negative control clones were negative.

Suitable dilutions of the final fd88FLAG culture supernatants were titered for blue plaques as described above. Of the plaques derived from clone $1,76 \%$ were blue and $24 \%$ white, and $9 \%$ of the clone 2 plaques were blue and $91 \%$ white (white plaques have lost all or part of the lac operator sometime during the six cycles of nonselective propagation, as explained in Results and Discussion). An NZY agar dish was seeded with 400 $\mu \mathrm{L}$ of an early log-phase NZY culture of K91 in $3 \mathrm{~mL}$ molten soft agar premixed with $60 \mu \mathrm{L} 2 \% \mathrm{X}$-gal. When the soft agar had gelled, 49 blue clone 1 plaques, 42 blue clone 2 plaques, and 11 white clone 2 plaques were picked to grid points on the seeded dish with sterile toothpicks. The gridded dish was then incubated at $37^{\circ} \mathrm{C}$ for about $20 \mathrm{~h}$ to allow the plaques to form and the blue color to develop. All blue primary plaques but none of the white primary plaques gave blue plaques on the gridded dish. The gridded dish was blotted with nitrocellulose, and the blot was washed, blocked, reacted, and developed as described above. None of the 11 white plaques turned out to be positive for FLAG in this test, and 26 of the 49 blue clone 1 plaques were positive, as were 37 of the 42 blue clone 2 plaques.

\section{RESULTS AND DISCUSSION}

\section{Properties of fd88-4 Vector and Clones f88-FLAG and fd88-FLAG}

When fd88-4 is plated for plaques in a wild-type $E$. coli host on nutrient agar containing X-gal but no IPTG (or lactose), the plaques are dark blue. The reason is that the lac operator on the high copy number phage genome (Figure 1) titrates the limited supply of Lac repressor molecules, thus derepressing the lac operon on the host-cell chromosome in the absence of IPTG or other lactose inducer. Subclones derived from fd88-4 that give white plaques in these circumstances have presumably lost all or part of the lac operator sometime during propagation. The fd88-4 display cassette (Figure 1) is also presumed to be fully derepressed, even if no IPTG is included in the medium. (Because of its low copy number, f88-4 does not derepress the host-cell lac operon or its own display cassette substantially in the absence of IPTG or other inducers.)

Four liters of fd88-4-infected cells grown to stationary phase yielded a total of $1.6 \times 10^{16}$ purified virions with an infectivity of $0.58 \mathrm{pfu} /$ particle and $2 \mathrm{mg}$ of purified supercoiled RF DNA. These statistics are typical for wild-type filamentous phage. The virion yield, infectivity, and RF yield are about 4, 10, and 2 times higher, respectively, than for fdtet-based phage. All plaques were blue, indicating that the incidence of phage that had lost the lac operator part of the gene VIII display cassette was very low.

The coding sequence for the FLAG peptide was ligated into the cloning sites of both fd88-4 and f88-4 as shown in the lower part of Figure 1, thus constructing the high copy number clone fd88-FLAG and the low copy number clone f88-FLAG. Similar to their respective parent vectors fd88-4 and f884, fd88-FLAG formed blue plaques and f88-FLAG formed tetracycline-resistant transductant colonies when titered as described above.

\section{Genetic Stability of the FLAG Peptide in f88-FLAG and fd88-FLAG}

Two clones each of the high copy number fd88-FLAG construct and the low copy number f88-FLAG construct were propagated through six cycles of nonselective propagation-that is, without regard to FLAG peptide expression-as described above. For all rounds of fd88-FLAG propagation and all but the first round of f88-FLAG propagation, the host cell was K91. IPTG was included to induce the expression of the display cassette during f88-FLAG propagation but was unnecessary during fd88-FLAG propagation. A random sample of a few dozen subclones from each of the four final phage populations was then tested for retention of the FLAG peptide and (in the case of fd88-FLAG) for a functional lac operator. Of the 98 f88-FLAG subclones tested, all but one were positive for the FLAG peptide. In contrast, only $76 \%$ and $8.8 \%$ of the subclones of fd88-FLAG clones 1 and 2, respectively, retained a functional lac operator, and of those that did, only $53 \%$ and $88 \%$, respectively, were positive for the FLAG peptide (as expected, none of the operator-negative subclones tested were positive for the FLAG peptide). Overall, therefore, $41 \%$ and $7.8 \%$ of the subclones derived from fd88-FLAG clones 1 and 2, respectively, retained the ability to express the FLAG peptide after six rounds of nonselective propagation. It is entirely possible that the observed differences between the clone 1 and clone 2 populations are merely stochastic, rather than the result of any intrinsic difference between their starting clones. In any case, there were no sequence differences in their gene VIII display cassettes.

There are at least two types of selective advantage an fd88-FLAG clone might gain by deleting all or part of its gene VIII display cassette. First, the DNA would be smaller and therefore able to replicate faster. However, no comparable advantage would accrue to an f88-FLAG clone because in that case it is initiation of minus-strand synthesis, and not completion of alreadyinitiated plus or minus strands, that is the rate-limiting step in replication. Second, expression of the recombinant pVIII would be eliminated, thus relieving the cell at the least of a slight metabolic burden and at the most of a slightly toxic protein (recall that the fd88-FLAG gene VIII display cassette 
is derepressed even in the absence of IPTG). In contrast, expression of the recombinant $p$ VIII from the f88-FLAG cassette, even when fully induced by IPTG, presumably imposes little metabolic burden or toxicity on the host cell because of the phage's low DNA copy number.

The instability observed in the high copy number fd88-FLAG phage is by no means so severe as to disqualify fd $88-4$ as a phage display vector. No loss of either functional lac operator or FLAG peptide expression was observed after the first cycle of nonselective propagation (data not shown). The cumulative loss after six cycles represents a very slow rate of loss that could easily be overcome by even a very modest selection in favor of a displayed peptide (e.g., by affinity selection with an immobilized target molecule). Indeed, high DNA copy number vectors, including commercially available vectors such as the Ph.D. ${ }^{\mathrm{TM}}$ system (New England Biolabs, Beverly, MA, USA), are used successfully in many applications. However, if-as seems entirely possible-long inserts are substantially more genetically unstable than the eight-residue FLAG peptide studied here, it may be prudent to use low DNA copy number vectors to display large peptides such as single-chain antibodies (about 240 amino acids).

\section{ACKNOWLEDGMENTS}

This work was supported by $\mathrm{Na}$ tional Institutes of Health grant no. GM41478 to G.P.S.

\section{REFERENCES}

1.Smith, G.P. and V.A. Petrenko. 1997. Phage display. Chem. Rev. 97:391-410.

2.Zinder, N.D. and K. Horiuchi. 1985. Multiregulatory element of filamentous bacteriophages. Microbiol. Rev. 49:101-106.

3.Kim, M.H., J.C. Hines, and D.S. Ray. 1981 Viable deletions of the M13 complementary strand origin. Proc. Natl. Acad. Sci. USA 78:6784-6788.

4.Zacher, A.N.I., C.A. Stock, J.W.I. Golden, and G.P. Smith. 1980. A new filamentous phage cloning vector: fd-tet. Gene 9:127-140.

5.Pratt, D., H. Tzagoloff, and W.S. Erdahl. 1966. Conditional lethal mutants of the small filamentous coliphage M13. I. Isolation, complementation, cell killing, time of cistron ac- tion. Virology 30:397-410.

6.Smith, G.P. 1988. Filamentous phage assembly: morphogenetically defective mutants that do not kill the host. Virology 167:156-165.

7.Zhong, G., G.P. Smith, J. Berry, and R.C. Brunham. 1994. Conformational mimicry of a chlamydial neutralization epitope on filamentous phage. J. Biol. Chem. 269:24183-24188.

8.Kouzmitcheva, G.A., V.A. Petrenko, and G.P. Smith. 2000. Identifying diagnostic peptides for lyme disease through epitope discovery. Clin. Diagn. Lab. Immunol. 8:150-160.

9.Matthews, L.J., R. Davis, and G.P. Smith. 2002. Immunogenically fit subunit vaccine components via epitope discovery from natural peptide libraries. J. Immunol. 169:837-846.
10.Yu, J. and G. Smith. 1996. Affinity maturation of phage-displayed peptide ligands. Methods Enzymol. 267:3-27.

Received 31 October 2003; accepted 20 January 2004.

Address correspondence to George P. Smith, Division of Biological Sciences, Tucker Hall, University of Missouri, Columbia, MO 65211,USA.e-mail: smithgp@missouri.edu

\title{
Comparison of mRNA gene expression by RT-PCR and DNA microarray
}

\author{
Wiguins Etienne' ${ }^{1}$, Martha H. Meyer ${ }^{1}$, Johnny Peppers ${ }^{2}$, and Ralph A. Meyer, Jr. ${ }^{1}$ \\ ${ }^{1}$ Carolinas Medical Center, Charlotte, NC and ${ }^{2}$ Affymetrix, Santa Clara, CA, USA
}

BioTechniques 36:618-626 (April 2004)

Few studies have compared the quantification of $m R N A$ by DNA microarray to the results obtained by reverse transcription PCR (RT-PCR). In this study, $m R N A$ was collected from the healing femoral fracture callus of adult and juvenile rats at various times after fracture. Ten samples were measured by both methods for 26 genes. For RT-PCR, mRNA was reverse transcribed, amplified, electrophoresed, blotted, and probed with ${ }^{32} P$-labeled internal oligonucleotides, which were quantified. For DNA microarray, the mRNA was processed to biotin-labeled cRNA, hybridized to 10 Affymetrix ${ }^{\circledR}$ Rat U34A microarrays, and quantified. Correlation coefficients $(r)$ for each gene for the agreement between RT-PCR and microarray ranged from -0.48 to +0.93 . This variation made the interpretation gene-specific. Genes with moderate expression levels gave the highest $\mathrm{r}$ values. Increased numbers of absent calls by the microarray software and increased separation between the location of the PCR primers and the microarray probes both led to reduced agreement. Microarray analysis suggested a floor effect in expression levels measured by RT-PCR for two genes. In conclusion, moderate $m R N A$ expression levels with overlap in the location of PCR primers and microarray probes can yield good agreement between these two methods.

\section{INTRODUCTION}

Messenger RNA (mRNA) can be quantified by a number of methods (1). Among the more popular methods for genes with relatively low expression levels has been reverse transcription PCR (RT-PCR). This semiquantitative method is labor-intensive and gives data for only one, or a very few, gene(s) for each assay (2). This has led to the search for more efficient methods for studying
mRNA levels for multiple genes.

DNA microarrays yield much greater data output since one hybridization results in the measurement of expression of all genes on the array at the same time (3). This allows the study of many gene transcripts of interest as well as the discovery of new genes involved in the system under study. While this new technology holds great promise, relatively few studies have been done to compare the results obtained by DNA microarray 